\title{
An Efficient Filtering Structure for Lagrange Interpolation
}

\author{
Çag̃atay Candan
}

\begin{abstract}
A novel filtering structure with linear complexity is proposed for Lagrange interpolation. The structure is similar to the Farrow structure in principle, but it is more efficient and has the additional feature of being order updatable on-the-fly. The main application for the proposed structure is the implementation of fractional delay filters to mitigate the symbol synchronization errors in digital communications. Some other applications are time-delay estimation, echo cancellation, acoustic modeling, and arbitrary sampling rate conversion.
\end{abstract}

Index Terms-Farrow structure, interpolation, Lagrange interpolation.

\section{INTRODUCTION}

CYNCHRONIZATION of system parameters at transmitting and receiving ends of a channel is critical to establish reliable communication. The mismatches in carrier frequency, carrier phase, or timing errors in signal sampling can significantly degrade communication. In this letter, we propose an efficient finite impulse response (FIR) interpolation structure that can be used in digital receivers to mitigate the symbol synchronization errors.

The proposed structure is an implementation for Lagrange interpolation. Lagrange interpolation is based on determining the $N$ th-order polynomial passing through $N+1$ sample points. Zero-order hold, linear, and cubic interpolation are some special cases of Lagrange interpolation. Lagrange interpolation has found applications in digital-to-analog converters, image processing, time-delay estimation, and communication problems.

In digital communications, the receiver clock may have timevarying offset causing intersymbol interference at the channel output. One way of compensating the error is to fractionally delay the signal [1], [2]. An efficient filtering structure for fractional delay generation is proposed by Farrow [3], [4]. The structure has an explicit parameter that can be adjusted during run time, and it is suitable for the applications whose delay parameter is frequently varied, as in synchronization or time-delay estimation problems. Farrow structure has been improved in [5], leading to some additional computational savings for low ordered interpolators. The structure has been extended to the filterbanks for wide-band signals. The Farrow filterbanks are optimal in the min-max error sense and designed to minimize the implementation cost [6], [7]. The hardware optimization of

Manuscript received February 13, 2006; revised June 2, 2006. The associate editor coordinating the review of this manuscript and approving it for publication was Dr. Hakan Johansson.

The author is with the Department of Electrical Engineering, Middle East Technical University, Ankara, Turkey (e-mail: ccandan@metu.edu.tr).

Digital Object Identifier 10.1109/LSP.2006.881528

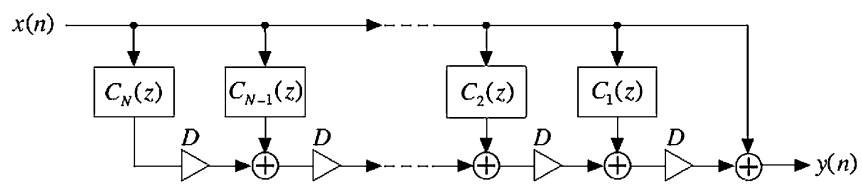

Fig. 1. Farrow structure.

Farrow structure [8] and several other applications on multichannel signal sampling and reconstruction have been discussed in [9]-[11].

In this letter, we present an alternative to the Farrow structure for Lagrange interpolation. The proposed structure has linearly growing computational complexity, while earlier proposals have complexities growing with the square of the interpolation order. Before the description of the proposed structure, we present a brief review of the Farrow structure with some of its extensions.

\section{FARROW STRUCTURE}

Farrow has proposed a filterbank structure with intermittent delay multipliers in [3]. The structure is shown in Fig. 1.

The interpolation output $Y(z)$ can be expressed as $Y(z)=$ $\sum_{k=0}^{N} C_{k}(z) D^{k} X(z)$, where $D$ is the adjustable delay parameter. For the structure to be interpolating, the output should be the delayed versions of the input for $D=\{0,1, \ldots, N\}$. This constraint imposes $N+1$ equations in the form $Y(z)=$ $z^{-D} X(z)$ for $D=\{0,1, \ldots, N\}$. The unknown $C_{k}(z)$ 's can be uniquely solved from these equations [3].

The computational needs of Farrow structure are $N^{2}+N$ multiplications and $N^{2}$ additions per output sample. Note that $C_{0}(z)$ is equal to 1 for all delay values; therefore, the implementation cost of $C_{0}(z)$ is discarded in this calculation. For low interpolation orders such as second or third, it is possible to customize the filter design by combining common multiplier factors together, resulting in some savings. However, in general, the computation requirements of the Farrow structure grows with the square of the interpolation order.

In [5], Farrow structure has been modified, and its computation load has been lightened by deleting the integer part of the delay parameter $D$. This has lead to some additional savings for low ordered interpolations. In [6], filterbanks approximating exact Lagrange interpolation in the min-max error sense is proposed. The computational cost of the filterbanks is further minimized in [7].

\section{Proposed Structure}

The proposed structure is based on discrete time Taylor series expansion. We briefly review the difference operators and factorial functions to illustrate the approach. 
The backward difference operator, $\Delta f[n]=f[n]-f[n-1]$, is the dual of derivative operator. Similarly, the discrete time dual of polynomial powers are called factorial polynomials and defined as follows:

$$
x^{[N]}=x(x+1)(x+2) \ldots(x+N-1) .
$$

As expected, when $\Delta$ is operated on the factorial polynomials, we get a factorial polynomial with one less degree, $\Delta x^{[N]}=$ $N x^{[N-1]}$. The discrete time dual of Taylor series can then be written in terms of factorial polynomials and difference operators

$$
\tilde{f}(t)=\sum_{n=0}^{\infty} \Delta^{n} f[k] \frac{(t-k)^{[n]}}{n !} .
$$

The implicit interpolation operation in (2) can be easily verified by substituting $t=k$ into the equation, leading to $\tilde{f}(t)=f[k]$. After the application of $\Delta$ to both sides of (2), if $t$ is substituted for $k$, we get $\Delta \tilde{f}(t)=\Delta f[k]$. By repeating the same operation for arbitrary powers of $\Delta$, we can show that $\tilde{f}(k-q)=f[k-q]$ for all $q$.

When the summation in (2) is truncated to a finite number of terms, sa,y $N$, the resultant relation is equivalent to fitting a $N$ th-degree polynomial to $N+1$ consecutive samples of $f[k]$. In other words, the truncated sum is the $N$ th-order Lagrange interpolation of the input.

The third-order interpolation relation can be explicitly written as

$$
\begin{aligned}
\tilde{f}(t)= & f[k]+\frac{\Delta f[k]}{1 !}(t-k)+\frac{\Delta^{2} f[k]}{2 !}(t-k)^{[2]} \\
& +\frac{\Delta^{3} f[k]}{3 !}(t-k)^{[3]} \\
= & f[k]+\frac{\Delta f[k]}{1 !}(-D)+\frac{\Delta^{2} f[k]}{2 !}(-D)^{[2]} \\
& +\frac{\Delta^{3} f[k]}{3 !}(-D)^{[3]}
\end{aligned}
$$

The output $\tilde{f}(t)$ denotes the interpolation result, and $D$ indicates the delay parameter as shown in Fig. 2.

The remarkable aspect of relation (3) is the simplicity of its implementation. The consecutive terms in summation (2) can be recursively calculated as follows:

$$
\begin{aligned}
\frac{(-D)^{[N]} \Delta^{N}}{N !} & f[k] \\
& =\frac{(-D)^{[N-1]} \Delta^{N-1}}{(N-1) !} \frac{(-D+N-1) \Delta}{N} f[k] .
\end{aligned}
$$

When the recursion is inserted in the Taylor summation, the overall structure simplifies to the structure shown in Fig. 3.

The computational complexity of the proposed structure is $3 N-2$ additions and $3 N-1$ multiplications for the $N$ th-order interpolation. Furthermore, once the multiplication factors for delay $D$ are calculated and stored, the overall complexity of delaying the next incoming sample for $D$ units reduces to $N$ multiplications and $2 N-2$ additions per output sample. In other

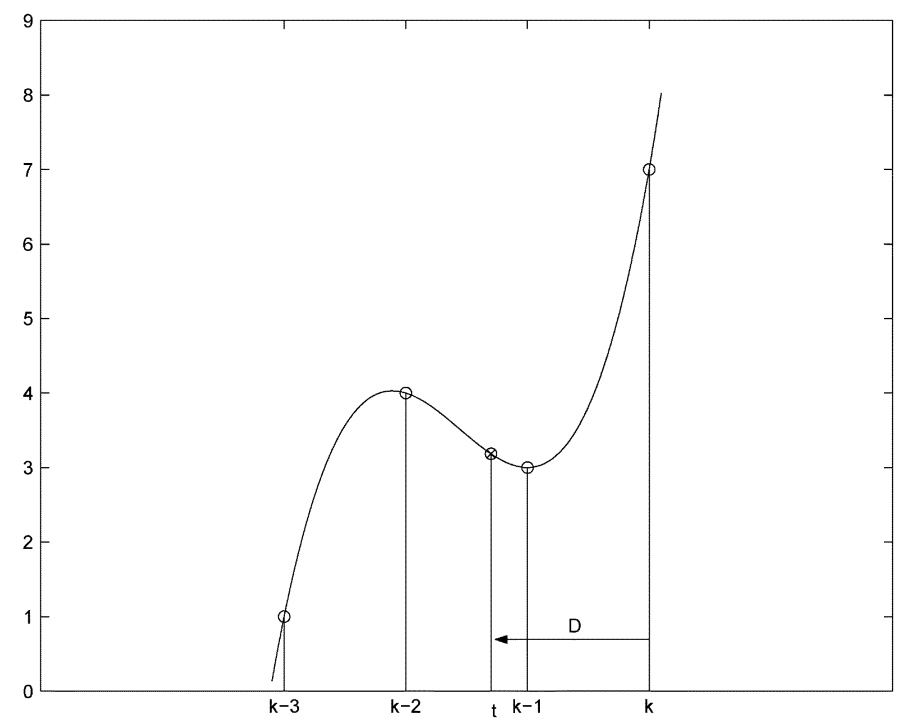

Fig. 2. Third-order interpolation.

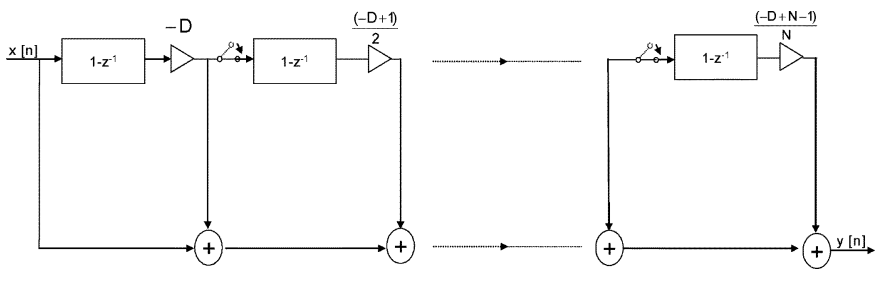

Fig. 3. Proposed structure.

TABLE I

COMPUTATIONAL COMPLEXITY COMPARISON FOR FARROW, MOdified FARROW, AND PROPOSED STRUCTURE. THE NUMBER of ADDitions AND MUlTiPlicATIONS ARE THE FIRST AND SECOND ELEMENTS OF EACH TABLE ENTRY

\begin{tabular}{l|l|l|l} 
Order & Farrow & M. Farrow & Proposed \\
\hline 1 & $(2,1)$ & $(2,1)$ & $(2,1)$ \\
2 & $(6,6)$ & $(4,4)$ & $(5,4)$ \\
3 & $(14,12)$ & $(11,9)$ & $(7,8)$ \\
$\mathrm{N}$ & $\left(O\left(N^{2}\right), O\left(N^{2}\right)\right)$ & $\left(O\left(N^{2}\right), O\left(N^{2}\right)\right)$ & $(3 N-2,3 N-1)$
\end{tabular}

words, computation load reduces to the level of $N$ tap FIR filtering with constant coefficients. In Table I, computational complexity requirements of different Lagrange interpolation implementations are compared.

A novel feature of the proposed structure is the run time increment-decrement possibility of the interpolation order. As shown in Fig. 3, the order of the structure can be easily changed by flipping the switch between the stages of filtering. This feature can be useful in applications requiring variable levels of interpolation as in software radio applications [12].

In Fig. 4, the magnitude and phase delay response of the 18th-order Lagrange interpolator is shown for different delay values. The worst-case magnitude and phase error in the frequency range $[0,(\pi / 2)]$ is 0.00049 and 0.00054 , respectively. Using the proposed structure, the implementation cost of the filter is reduced from $18^{2}$ multiplications to 18 multiplications with stored coefficients and to 53 coefficients with calculated 

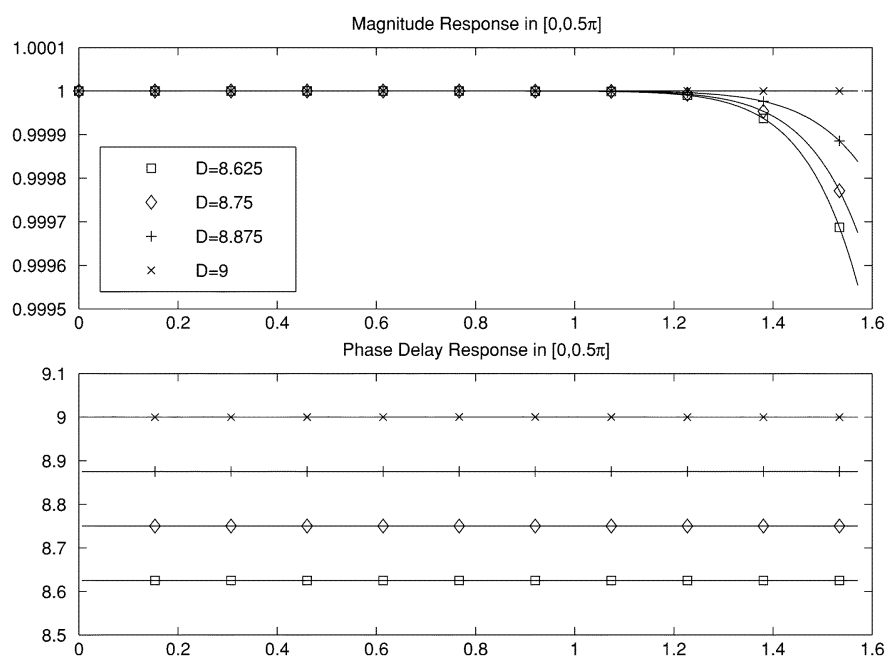

Fig. 4. Magnitude and phase delay response of 18th-order Lagrange interpolator.

coefficients. In [7, Ex. 3], a filterbank is optimized to generate fractional delays with the maximum magnitude and phase error of 0.001 . The resulting optimized filterbank requires 19 multiplications per output sample. When two systems are compared, we note that the standard Lagrangian filter, which does not require any optimization, becomes competitive with the optimized one when implemented with the proposed structure. In other words, the maximally flat Lagrange interpolators [2], whose frequency response closely approximates the ideal delay response for low bandwidth signals, can be implemented at reduced costs. Therefore, higher order Lagrange filters needed for higher bandwidth inputs can be cost efficiently implemented with the proposed structure, leading to a leverage between the optimized designs of [7] and standard Lagrangian filters.

\section{CONCLUSION}

We have presented an efficient structure for Lagrange interpolation. The structure is well suited for the applications requiring frequent variation of the interpolation parameter. The proposed structure has linearly growing computational complexity, while alternative structures have complexities growing with the square of the interpolation order. We believe that such a reduction in complexity can be critical in synchronization or time-delay estimation applications. Additionally, the interpolation order of the proposed structure can be changed during run time, leading to a useful feature for applications such as software defined radio applications, which heavily depend on interpolation for sampling rate conversion.

\section{REFERENCES}

[1] L. Erup, F. M. Gardner, and R. A. Harris, "Interpolation in digital modems. II. Implementation and performance," IEEE Trans. Commun., vol. 41, no. 6, pp. 998-1008, Jun. 1993.

[2] S. Samadi, M. O. Ahmad, and M. N. S. Swamy, "Results on maximally flat fractional-delay systems," IEEE Trans. Circuits Syst. I, vol. 51, no. 11, pp. 2271-2286, Nov. 2004

[3] C. W. Farrow, "A continuously variable digital delay element," in Proc. IEEE Int. Symp. Circuits Systems, 1988, pp. 2641-2645.

[4] T. Laakso, V. Valimaki, M. Karjalainen, and U. Laine, "Splitting the unit delay," IEEE Signal Process. Mag., vol. 13, no. 1, pp. 30-60, Jan. 1996.

[5] V. Valimaki, "A new filter implementation strategy for Lagrange interpolation," in Proc. IEEE Int. Symp. Circuits Systems, 1995, pp. 361-364.

[6] J. Vesma and T. Saramäki, "Optimization and efficient implementation of FIR filters with adjustable fractional delay," in Proc. IEEE Int. Conf. Acoustics, Speech, Signal Processing, 1997, pp. 2256-2259.

[7] H. Johansson and P. Löwenborg, "On the design of adjustable fractional delay FIR filters," IEEE Trans. Circuits Syst. II, vol. 50, no. 4, pp. 164-169, Apr. 2003.

[8] C. Pun, Y. Wu, S. Chan, and K. Ho, "On the design and efficient implementation of the Farrow structure," IEEE Signal Process. Lett., vol. 10, no. 7, pp. 189-192, Jul. 2003.

[9] D. Luengo, C. Pantaleon, J. Ibanez, and I. Santamaria, "Design of simultaneous sampling systems based on fractional delay Lagrange filters," IEEE Trans. Circuits Syst. II, vol. 47, no. 5, pp. 482-485, May 2000.

[10] A. G. Dempster and N. P. Murphy, "Efficient interpolators and filter banks using multiplier blocks," IEEE Trans. Signal Process., vol. 48, no. 1, pp. 257-261, Jan. 2000.

[11] H. Johansson and O. Gustafsson, "Linear-phase FIR interpolation, decimation, and $m$ th-band filters utilizing the Farrow structure," IEEE Trans. Circuits Syst. I, vol. 52, no. 10, pp. 2197-2207, Oct. 2005.

[12] W. Abu-Al-Saud and G. Stuber, "Modified CIC filter for sample rate conversion in software radio systems," IEEE Signal Process. Lett., vol. 10 , no. 5, pp. 152-154, May 2003. 